\title{
The systemic immune-inflammation index-based model is an effective biomarker on predicting central lymph node metastasis in clinically nodal-negative papillary thyroid carcinoma
}

\author{
Zeyu Zhang ${ }^{1}$, Fada Xia ${ }^{1}$, Wenlong Wang ${ }^{1}$, Yun Huang ${ }^{2}$, Xinying $\mathrm{Li}^{1}$ \\ ${ }^{1}$ Department of Thyroid Surgery, Xiangya Hospital, Central South University, Changsha, China; ${ }^{2}$ Department of General Surgery, Xiangya Hospital, \\ Central South University, Changsha, China \\ Contributions: (I) Conception and design: X Li, Y Huang, F Xia; (II) Administrative support: X Li, F Xia; (III) Provision of study materials or patients: \\ Z Zhang, W Wang, F Xia; (IV) Collection and assembly of data: Z Zhang, W Wang, F Xia, Y Huang; (V) Data analysis and interpretation: Z Zhang, \\ W Wang, F Xia, X Li; (VI) Manuscript writing: All authors; (VII) Final approval of manuscript: All authors. \\ Correspondence to: Xinying Li, PhD; Fada Xia, PhD. Department of Thyroid Surgery, Xiangya Hospital, Central South University, No. 87 Xiangya \\ Road, Changsha, China. Email: lixinyingcn@126.com; xiafada@csu.edu.cn.
}

\begin{abstract}
Background A high proportion of papillary thyroid carcinoma (PTC) patients are involved in central lymph node metastasis (CLNM) with preoperative imaging examinations showing clinically nodal-negative (cN0). Meanwhile, many inflammatory biomarkers are also proven as effective factors to predict the outcomes of cancer patients and tumor progression. Thus, the values of these factors are investigated to help detecting CLNM in cN0 PTC patients.
\end{abstract}

Methods: 406 cN0 PTC patients who underwent curative surgery were retrospectively analyzed. CLNM was determined by histopathological examination following the thyroidectomy. Multiple inflammatory biomarkers were comprehensively researched.

Results: A total of 406 consecutive patients were eventually included. The univariate and multivariate analyses revealed that age (OR: 0.924, 95\% CI: 0.909, 0.940), gender (OR: 1.781, 95\% CI: 1.060, 2.993), location of tumors (OR: 2.229, 95\% CI: 1.228, 4.046) and level of systemic immune-inflammation index (SII) (OR: 1.005, 95\% CI: 1.004, 1.006) were independently associated with CLNM in cN0 PTC patients, and the SII-based predictive model was constructed using these four factors. Receiver operating characteristic (ROC) curves showed significant results of the SII-based predictive model in PTC cohort with area under curve (AUC) as 0.814 (95\% CI: 0.771-0.857) and in PTMC subgroup with AUC as 0.803 (95\% CI: 0.752$0.854)$.

Conclusions: The SII-based model can effectively help predicting CLNM in both cN0 PTC patients and cN0 PTMC patients.

Keywords: Papillary thyroid carcinoma (PTC); central lymph node metastasis (CLNM); inflammatory biomarker; systemic immune-inflammation index (SII)

Submitted Aug 14, 2020. Accepted for publication Feb 04, 2021.

doi: $10.21037 /$ gs-20-666

View this article at: http://dx.doi.org/10.21037/gs-20-666

\section{Introduction}

Papillary thyroid carcinoma (PTC) is the most common thyroid malignancy with a growing worldwide incidence in the last decade (1). PTC usually carries a good patient prognosis. However, 30-80\% PTC patients are involved in lymph node metastasis (LNM) including central lymph node metastasis (CLNM) and lateral lymph node metastasis, which is considered as a significant prognostic factor in PTC $(2,3)$. Currently, preoperative evaluations of LNM mainly focus on imaging examinations such as ultrasound 
and computed tomography. However, there is a considerable number of patients with CLNM where preoperative imaging examinations show clinically nodal-negative (cN0) because of false-negative diagnosis of imaging examination, which may disturb the medical decision-making (4). Thus, other tools in addition to imaging examinations are needed to help detecting CLNM, therefore can contribute to a better preoperative medical decision.

Inflammation is proven to be able to promote proliferation, angiogenesis and metastasis of cancer tissues via multiple mechanisms and pathways by increasing evidences (5). Many inflammatory biomarkers, including neutrophil-tolymphocyte ratio (NLR), platelet-to-lymphocyte ratio (PLR), lymphocyte-to-monocyte ratio (LMR), systemic immuneinflammation index (SII), are also proven as effective factors to predict the outcomes of cancer patients and tumor progression (6-8). But the associations between these inflammatory biomarkers and PTC are poorly investigated.

In this study, we aimed at investigating the significance of inflammatory biomarkers on predicting CLNM in clinically nodal-negative (cN0) PTC patients. Additionally, a subgroup analysis was also performed among patients with papillary thyroid microcarcinoma (PTMC). We presented the following article in accordance with the REMARK reporting checklist (available at http://dx.doi.org/10.21037/gs-20-666).

\section{Methods}

\section{Study cobort}

Patients who were diagnosed as PTC and underwent surgery in Xiangya Hospital, Central South University from February 2019 to January 2020 were retrospectively analyzed. The exclusion criteria were as follows: (I) PTC was not pathological confirmed; (II) recurrent PTC; (III) preoperative examinations showed signs or suspicious signs of LNM (cN0); (IV) suffering from infectious diseases, immunodeficiency diseases or autoimmune diseases including Hashimoto thyroiditis; (V) incomplete clinical data. The study was conducted in accordance with the Declaration of Helsinki (as revised in 2013). The study was approved by the Ethics Committee of Xiangya Hospital Central South University (No. 201912454) and individual consent for this retrospective analysis was waived.

\section{Definitions and surgical strategy}

TNM stage of PTC was determined using the 8th TNM staging system. NLR and PLR were determined as neutrophil count/lymphocyte count and platelet count/ lymphocyte count, respectively. LMR and SII were determined as lymphocyte count/monocyte count and platelet count $\times$ neutrophil count/lymphocyte count, respectively. PTC with maximum tumor diameter $\leq 10 \mathrm{~mm}$ was considered as PTMC.

Ultrasound and enhanced computed tomography were used to preoperatively assess LNM, while magnetic resonance imaging was used as appropriate. Bilateral or unilateral prophylactic central lymph node dissection was routinely performed in either total thyroidectomy or hemithyroidectomy when intraoperative frozen pathology confirmed the nodules as PTC. CLNM was finally determined by histopathological examinations.

\section{Statistical analysis}

Statistical analyses and data visualization were realized by SPSS 22.0 and Prism software. Quantitative data were expressed as mean \pm standard deviation (SD) and analyzed by independent-sample $t$-test, while categorical data were expressed as frequency (percentage) and analyzed by Chi-square or Fisher exact test. Receiver operating characteristic (ROC) curves, area under curve (AUC) and Youden index were used to investigate the value of inflammatory biomarkers-based models in predicting LNM and to determine the cutoff values. Meanwhile, the logistic regression was performed to investigate risk factors of CLNM, and factors with $\mathrm{P} \leq 0.10$ in univariate analyses were included in multivariate analyses. The formula of predictive model was determined by the regression coefficient ( $\beta$ ) of each factor which was adjusted in multivariate analyses. Statistical significance was recognized when $\mathrm{P} \leq 0.05$.

The study was conducted in accordance with the Declaration of Helsinki (as revised in 2013). The study was approved by the Ethics Committee of Xiangya Hospital Central South University (No. 201912454) and individual consent for this retrospective analysis was waived.

\section{Results}

\section{Cohort and tumor characteristics}

As shown in Table 1, 406 patients were finally included in this study with the mean age as 39.95 years old. Of them 107 were male while 299 were female. One hundred twenty patients suffered from multifocal tumors and 
Table 1 Clinicopathologic variables of PTC patients

\begin{tabular}{|c|c|}
\hline Variables & Values $(n=406)$ \\
\hline Age (years) & $39.95 \pm 9.50$ \\
\hline Male & $107(26.4)$ \\
\hline Tumor size (cm) & $0.85 \pm 0.57$ \\
\hline \multicolumn{2}{|l|}{ Number of tumors } \\
\hline Single & $286(70.4)$ \\
\hline Multiple & $120(29.6)$ \\
\hline Bilateral tumors & 75 (18.5) \\
\hline \multicolumn{2}{|c|}{ T stage (tumor extension) } \\
\hline I & 391 (96.3) \\
\hline II & $14(3.5)$ \\
\hline III & $1(0.2)$ \\
\hline \multicolumn{2}{|l|}{ Capsule invasion } \\
\hline No & 362 (89.2) \\
\hline Yes & $44(10.8)$ \\
\hline \multicolumn{2}{|l|}{ CLNM } \\
\hline No & $246(60.6)$ \\
\hline Yes & $160(39.4)$ \\
\hline Neutrophil $\left(10^{9} / \mathrm{L}\right)$ & $3.57 \pm 1.18$ \\
\hline Lymphocyte $\left(10^{9} / \mathrm{L}\right)$ & $1.90 \pm 0.55$ \\
\hline Monocyte $\left(10^{9} / \mathrm{L}\right)$ & $0.43 \pm 0.13$ \\
\hline $\operatorname{PLT}\left(10^{9} / \mathrm{L}\right)$ & $227.67 \pm 63.71$ \\
\hline NLR & $2.02 \pm 0.86$ \\
\hline PLR & $126.92 \pm 41.24$ \\
\hline LMR & $4.65 \pm 1.60$ \\
\hline SII & $461.20 \pm 251.66$ \\
\hline
\end{tabular}

Data are expressed as mean \pm standard deviation or $\mathrm{n}(\%)$. PTC, papillary thyroid carcinoma; CLNM, central lymph node metastasis; PLT, Blood platelet; NLR, neutrophil-to-lymphocyte ratio; PLR, platelet-to-lymphocyte ratio; LMR, lymphocyte-tomonocyte ratio; SII, systemic immune-inflammation index.

75 of them suffered from bilateral tumors. The mean tumor size was $0.85 \mathrm{~cm}$. Most of the patients were found as T1 stage $(n=391,96.3 \%)$ and 44 patients were found as having capsule invasion. The mean values of neutrophil, lymphocyte, monocyte and platelet counts were $(3.57 \pm 1.18) \times 10^{9} / \mathrm{L},(1.90 \pm 0.55) \times 10^{9} / \mathrm{L},(0.43 \pm 0.13) \times 10^{9} / \mathrm{L}$ and $(227.67 \pm 63.71) \times 10^{9} / \mathrm{L}$, while NLR, PLR, LMR and SII were $2.02 \pm 0.86,126.92 \pm 41.24,4.65 \pm 1.60$ and $461.20 \pm 251.66$, respectively. One hundred sixty patients with CLNM were finally confirmed by histopathology.

\section{Univariate and multivariate analyses of CLNM}

To investigate the predictive value of NLR, PLR, LMR and SII on CLNM and create a predictive model of CLNM, univariate and multivariate analyses were subsequently performed (Table 2). Age (OR: 0.924, 95\% CI: 0.909, 0.940), gender (OR: 1.781, 95\% CI: 1.060, 2.993), location of tumors (OR: 2.229, 95\% CI: 1.228, 4.046) and level of SII (OR: 1.005 , 95\% CI: 1.004, 1.006) were the independent risk factors of CLNM in cN0 PTC patients. According to coefficient of each factor, the predictive model was finally determined as follow: predictive model $=0.577 \times(0$ for female, 1 for male $)-0.079 \times$ age (years $)+0.802 \times(0$ for unilateral, 1 for bilateral $)+0.005 \times$ SII. The mean value of the predictive model in this study was $-0.55 \pm 1.59$.

\section{Values of the SII-based predictive model on predicting CLNM}

To confirm the efficacy of the SII-based predictive model on predicting CLNM, ROC curves in the whole PTC cohort and the PTMC subgroup were respectively created, (Figure 1). Statistically significant results were shown in both the whole PTC cohort $(\mathrm{P}<0.001)$ and the PTMC subgroup $(\mathrm{P}<0.001)$. Furthermore, the predictive model had a better effectiveness in the whole PTC cohort with AUC as 0.814 (95\% CI: 0.771-0.857) than in the PTMC subgroup with AUC as 0.803 (95\% CI: 0.752-0.854). Meanwhile, the ideal cutoff values of the SII-based predictive model in PTC and PTMC were determined as -0.5463 and -0.3198 based on Youden index (https://cdn.amegroups.cn/static/ public/10.21037gs-20-666-1.xlsx).

\section{Discussion}

LNM is commonly seen in PTC and associated with the recurrence and survival of PTC patients. Meanwhile, there are increased risks of postoperative complications and quality of life when it comes to reoperation of PTC (9). Therefore, a preoperative prediction of CLNM can benefit cN0 PTC patients. In this study, we investigated multiple inflammatory biomarkers, and created a predictive model to predict CLNM in PTC patients. The results showed that the SII-based predictive model could effectively 
Table 2 Univariate and multivariate analyses of risk factors on CLNM in PTC patients

\begin{tabular}{|c|c|c|c|}
\hline \multirow{2}{*}{ Variables } & \multirow{2}{*}{$\begin{array}{c}\text { Number of } \\
\text { patients }\end{array}$} & \multicolumn{2}{|l|}{ CLNM } \\
\hline & & OR $(95 \% \mathrm{Cl})$ & $P$ value \\
\hline \multicolumn{4}{|l|}{ Univariate analyses } \\
\hline Age (years) & & $0.951(0.930,0.972)$ & $<0.001$ \\
\hline $\begin{array}{l}\text { Gender (male vs. } \\
\text { female) }\end{array}$ & 107 vs. 299 & $1.672(1.070,2.613)$ & 0.024 \\
\hline $\begin{array}{l}\text { Number of tumors } \\
\text { (multiple vs. single) }\end{array}$ & 120 vs. 286 & $1.200(0.778,1.852)$ & 0.409 \\
\hline $\begin{array}{l}\text { Location of tumors } \\
\text { (bilateral vs. } \\
\text { unilateral) }\end{array}$ & 75 vs. 331 & $1.647(0.994,2.727)$ & 0.053 \\
\hline $\begin{array}{l}\text { T stage (III and II } \\
\text { vs. I) }\end{array}$ & 15 vs. 391 & $1.026(0.358,2.940)$ & 0.962 \\
\hline $\begin{array}{l}\text { Capsule invasion } \\
\text { (presence vs. } \\
\text { absence) }\end{array}$ & 44 vs. 362 & $0.683(0.364,1.280)$ & 0.234 \\
\hline NLR & & $3.474(2.489,4.848)$ & $<0.001$ \\
\hline PLR & & $1.018(1.012,1.024)$ & $<0.001$ \\
\hline LMR & & $0.941(0.830,1.068)$ & 0.348 \\
\hline SII & & $1.006(1.004,1.007)$ & $<0.001$ \\
\hline \multicolumn{4}{|l|}{ Multivariate analyses } \\
\hline Age (years) & & $0.924(0.909,0.940)$ & $<0.001$ \\
\hline $\begin{array}{l}\text { Gender (male vs. } \\
\text { female) }\end{array}$ & & $1.781(1.060,2.993)$ & 0.029 \\
\hline $\begin{array}{l}\text { Location of tumors } \\
\text { (bilateral vs. } \\
\text { unilateral) }\end{array}$ & & $2.229(1.228,4.046)$ & 0.008 \\
\hline NLR & & & 0.066 \\
\hline PLR & & & 0.310 \\
\hline SII & & $1.005(1.004,1.006)$ & $<0.001$ \\
\hline
\end{tabular}

CLNM, central lymph node metastasis; PTC, papillary thyroid carcinoma; OR, odds ratio; $\mathrm{Cl}$, confidence interval; NLR, neutrophil-to-lymphocyte ratio; PLR, platelet-to-lymphocyte ratio; LMR, Iymphocyte-to-monocyte ratio; SII, systemic immune-inflammation index.

predict CLNM in both cN0 PTC patients and cN0 PTMC patients.

Neutrophils, as a subtype of leukocyte, serves as the crucial part of innate immunity which are able to eliminate pathogens by recruitment (10). It not only represents acute inflammation such as infections, but also participates in some complex physiological and pathological processes including carcinogenesis (11). Growing evidences indicate that neutrophils which can be recruited into tumor tissues via CXC chemokine ligands, interleukin and tumour necrosis factor, are associated with tumor growth, progression and metastasis by secreting relevant cytokines and growth factors, suppressing innate and adaptive immunity (12). Meanwhile, platelets can secrete TGF-beta, VEGF and platelet derived growth factors, which plays an emerging role in tumor progression and metastasis (13). There are also evidences supporting that platelets promote tumor metastasis by preparing the metastatic microenvironment, promoting neovasculature and impairing the defense mechanisms (14). On the contrary, lymphocytes are mainly recognized as a suppressor of tumor progression by promoting cytotoxic activity of macrophages and enhancing the expression of immune modulatory molecules on tumor cells (15). Furthermore, cancer immunotherapy focusing on $\mathrm{T}$ cells has emerged as a powerful tool against cancer (16).

Previous studies also focused on the value of inflammatory biomarkers in PTC patients. A few studies showed that preoperative levels of NLR and PLR were associated with poorer survival and recurrence data of PTC $(17,18)$. There were also some studies showing that PTC patients with a lower LMR had a poorer prognosis and a higher risk of recurrence (19-21). Besides, advanced tumor stage of PTC was found to be associated with a higher NLR and a lower LMR (22-24). However, few studies investigated the associations between these biomarkers and CLNM in cN0 PTC patients. One study by Lang et al. suggested that NLR was not associated with the risk of CLNM in cN0 PTC patients, which was inconsistent with our results (25). Our study included patients undergone bilateral prophylactic central lymph node dissection, and more importantly, performed ROC curves to determine the value of the predictive model. Nevertheless, our results did indicate limited significance of NLR in predicting CLNM in cN0 PTC patients. As far as we know, this was the first study investigating the value of SII and creating a predictive model in PTC patients. Currently, prophylactic central lymph node dissection was recommended among PTC patients. However, only few cN0 PTC would develop apparent lymph node metastasis during a long-term followup (26). Our results could help to distinguish non-CLNM PTC patients from CLNM PTC patients, thus, avoiding unnecessary prophylactic central lymph node dissection.

Nevertheless, limitations still existed with the present 

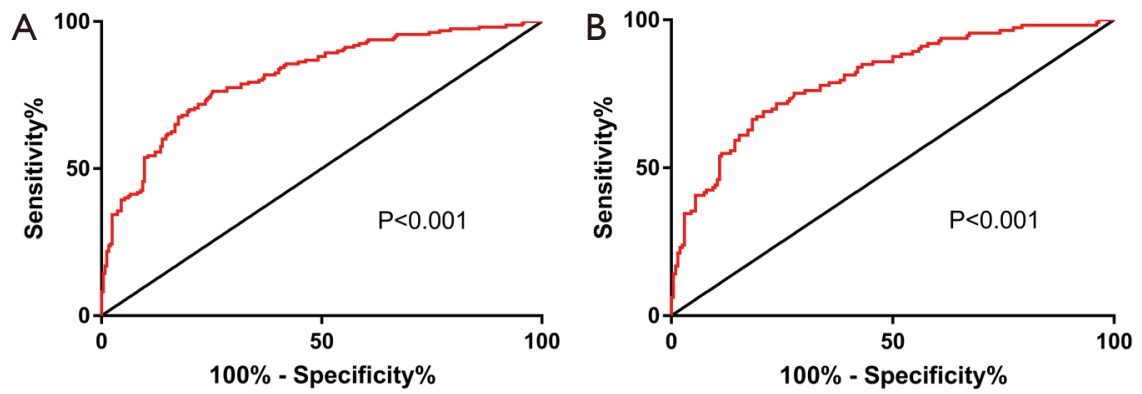

Figure 1 ROC curves of the SII-based predictive model on CLNM in cN0 PTC patients (A) and cN0 PTMC patients (B). (A) The AUC was 0.814 (95\% CI: 0.771-0.857, $\mathrm{P}<0.001$ ); (B) the AUC was 0.803 (95\% CI: 0.752-0.854, $\mathrm{P}<0.001$ ). ROC, receiver operating characteristic; AUC, area under curve; SII, systemic immune-inflammation index; CLNM, central lymph node metastasis; PTC, papillary thyroid carcinoma.

study. Firstly, a few problems lay in its retrospective design. Further prospective studies could be expected to confirm our results. Furthermore, the level of neutrophil, lymphocyte, platelet and monocyte was determined by a single test when admission, which could lead to bias. Lastly, the preoperative detection of CLNM using imaging examinations might make mistakes.

\section{Conclusions}

In short, the present study suggests that SII-based predictive model can effectively predict CLNM in both cN0 PTC patients and $\mathrm{cN} 0$ PTMC patients.

\section{Acknowledgments}

Funding: This work was supported by the National Natural Science Foundation of China (grant No. 81672885) and the Hunan Province Natural Science Foundation (grant number 2019JJ40475).

\section{Footnote}

Reporting Checklist: The authors have completed the REMARK reporting checklist. Available at http://dx.doi. org/10.21037/gs-20-666

Data Sharing Statement: Available at http://dx.doi. org/10.21037/gs-20-666

Conflicts of Interest: All authors have completed the ICMJE uniform disclosure form. XL serves as an unpaid associate editor of Gland Surgery from March 2018 to February 2023.
The other authors have no conflicts of interest to declare.

Ethical Statement: The authors are accountable for all aspects of the work in ensuring that questions related to the accuracy or integrity of any part of the work are appropriately investigated and resolved. The study was conducted in accordance with the Declaration of Helsinki (as revised in 2013). The study was approved by the Ethics Committee of Xiangya Hospital Central South University (No. 201912454) and individual consent for this retrospective analysis was waived.

Open Access Statement: This is an Open Access article distributed in accordance with the Creative Commons Attribution-NonCommercial-NoDerivs 4.0 International License (CC BY-NC-ND 4.0), which permits the noncommercial replication and distribution of the article with the strict proviso that no changes or edits are made and the original work is properly cited (including links to both the formal publication through the relevant DOI and the license). See: https://creativecommons.org/licenses/by-nc-nd/4.0/.

\section{References}

1. Steuer CE, El-Deiry M, Parks JR, et al. An update on larynx cancer. CA Cancer J Clin 2017;67:31-50.

2. Kluijfhout WP, Drake FT, Pasternak JD, et al. Incidental positive lymph nodes in patients with papillary thyroid cancer is independently associated with recurrent disease. J Surg Oncol 2017;116:275-80.

3. Zheng G, Zhang H, Hao S, et al. Patterns and clinical significance of cervical lymph node metastasis in papillary thyroid cancer patients with Delphian lymph node 
metastasis. Oncotarget 2017;8:57089-98.

4. Yang Z, Heng Y, Lin J, et al. Nomogram for Predicting Central Lymph Node Metastasis in Papillary Thyroid Cancer: A Retrospective Cohort Study of Two Clinical Centers. Cancer Res Treat 2020;52:1010-8.

5. Mantovani A, Allavena P, Sica A, et al. Cancer-related inflammation. Nature 2008;454:436-44.

6. Choi ES, Kim HS, Han I. Elevated preoperative systemic inflammatory markers predict poor outcome in localized soft tissue sarcoma. Ann Surg Oncol 2014;21:778-85.

7. Russo A, Russano M, Franchina T, et al. Neutrophil-toLymphocyte Ratio (NLR), Platelet-to-Lymphocyte Ratio (PLR), and Outcomes with Nivolumab in Pretreated NonSmall Cell Lung Cancer (NSCLC): A Large Retrospective Multicenter Study. Adv Ther 2020;37:1145-55.

8. Wang D, Bai N, Hu X, et al. Preoperative inflammatory markers of NLR and PLR as indicators of poor prognosis in resectable HCC. Peerj 2019;7:e7132.

9. Zhang C, Li X, Zhang Z, et al. The potential role of carbon nanoparticles-assisted biopsy for sentinel lymph nodes of incidental thyroid carcinoma. Gland Surg 2019;8:370-7.

10. Kolaczkowska E, Kubes P. Neutrophil recruitment and function in health and inflammation. Nature reviews. Immunology 2013;13:159-75.

11. Jaillon S, Galdiero MR, Del Prete D, et al. Neutrophils in innate and adaptive immunity. Semin Immunopathol 2013;35:377-94.

12. Jaillon S, Ponzetta A, Di Mitri D, et al. Neutrophil diversity and plasticity in tumour progression and therapy. Nat Rev Cancer 2020;20:485-503.

13. Qian H, Appiah-Kubi K, Wang Y, et al. The clinical significance of platelet-derived growth factors (PDGFs) and their receptors (PDGFRs) in gastric cancer: A systematic review and meta-analysis. Crit Rev Oncol Hematol 2018;127:15-28.

14. Gkolfinopoulos S, Jones RL, Constantinidou A. The Emerging Role of Platelets in the Formation of the Micrometastatic Niche: Current Evidence and Future Perspectives. Front Oncol 2020;10:374.

15. Seliger B. The Role of the Lymphocyte Functional Crosstalk and Regulation in the Context of Checkpoint Inhibitor Treatment-Review. Front Immunol 2019;10:2043.

16. Waldman AD, Fritz JM, Lenardo MJ. A guide to cancer immunotherapy: from $\mathrm{T}$ cell basic science to clinical practice. Nat Rev Immunol 2020;20:651-68.
17. Chen W, Wei T, Li Z, et al. Association of the Preoperative Inflammation-Based Scores with TNM Stage and Recurrence in Patients with Papillary Thyroid Carcinoma: A Retrospective, Multicenter Analysis. Cancer Manag Res 2020;12:1809-18.

18. Feng J, Wang Y, Shan G, et al. Clinical and prognostic value of neutrophil-lymphocyte ratio for patients with thyroid cancer: A meta-analysis. Medicine 2020;99:e19686.

19. Song L, Zhu J, Li Z, et al. The prognostic value of the lymphocyte-to-monocyte ratio for high-risk papillary thyroid carcinoma. Cancer Manag Res 2019;11:8451-62.

20. Ahn J, Song E, Kim WG, et al. Prognostic role of the lymphocyte-to-monocyte ratio for clinical outcomes of patients with progressive radioiodine-refractory differentiated thyroid carcinoma treated by sorafenib. Clin Endocrinol (Oxf) 2020;92:71-6.

21. Yokota M, Katoh H, Nishimiya H, et al. LymphocyteMonocyte Ratio Significantly Predicts Recurrence in Papillary Thyroid Cancer. J Surg Res 2020;246:535-43.

22. Ceylan Y, Kumanlıoğlu K, Oral A, et al. The Correlation of Clinicopathological Findings and Neutrophil-toLymphocyte and Platelet-to-Lymphocyte Ratios in Papillary Thyroid Carcinoma. Mol Imaging Radionucl Ther 2019;28:15-20.

23. Gong $W$, Yang S, Yang X, et al. Blood preoperative neutrophil-to-lymphocyte ratio is correlated with TNM stage in patients with papillary thyroid cancer. Clinics (Sao Paulo) 2016;71:311-4.

24. Wen W, Wu P, Li J, et al. Predictive values of the selected inflammatory index in elderly patients with papillary thyroid cancer. J Transl Med 2018;16:261.

25. Lang BH, $\mathrm{Ng} \mathrm{CP}, \mathrm{Au} \mathrm{KB}$, et al. Does preoperative neutrophil lymphocyte ratio predict risk of recurrence and occult central nodal metastasis in papillary thyroid carcinoma? World J Surg 2014;38:2605-12.

26. Sugitani I, Toda K, Yamada K, et al. Three distinctly different kinds of papillary thyroid microcarcinoma should be recognized: our treatment strategies and outcomes. World J Surg 2010;34:1222-31.

Cite this article as: Zhang Z, Xia F, Wang W, Huang Y, Li X. The systemic immune-inflammation index-based model is an effective biomarker on predicting central lymph node metastasis in clinically nodal-negative papillary thyroid carcinoma. Gland Surg 2021;10(4):1368-1373. doi: 10.21037/gs-20-666 